\title{
Methanol extract of Guettarda speciosa Linn. inhibits the production of inflammatory mediators through the inactivation of Syk and JNK in macrophages
}

\author{
HIEN THI THU LE ${ }^{1 *}$, YOUNG-CHANG CHO ${ }^{1,2^{*}}$ and SAYEON CHO ${ }^{1}$ \\ ${ }^{1}$ Laboratory of Molecular Pharmacological Cell Biology, College of Pharmacy, \\ Chung-Ang University, Seoul 06974, Republic of Korea
}

Received August 14, 2017; Accepted January 5, 2018

DOI: $10.3892 /$ ijmm.2018.3377

\begin{abstract}
Guettarda speciosa Linn. (G. speciosa, Rubiaceae) has been used as a traditional medicinal plant in Asia for the treatment of various inflammatory conditions, including cough, fever and maternal postpartum infection. However, the mechanisms underlying the anti-inflammatory action of $G$. speciosa extracts have remained elusive. In the present study, the anti-inflammatory effects of the methanol extract of G. speciosa (MGS) were investigated in murine macrophages by measuring the production of inflammatory mediators and the underlying mechanisms of action by performing immunoblotting analysis of proteins that are potentially involved. MGS reduced nitric oxide (NO) production through regulation of the expression of inducible NO synthase (iNOS) in lipopolysaccharide-activated RAW 264.7 cells; however, cyclooxygenase-2, the enzyme responsible for prostaglandin $\mathrm{E}_{2}$ production, was not affected at the mRNA or protein level. MGS reduced interleukin-6 (IL-6) production, but had no effect on tumor necrosis factor (TNF)- $\alpha$ production. In addition, MGS suppressed the transcription of IL-6, but not that of IL-1 $\beta$ and TNF- $\alpha$. The effect of MGS on proinflammatory mediators resulted from the inhibition of the activation of spleen tyrosine kinase and c-Jun N-terminal kinase. In conclusion, the present study suggested that MGS may be a potential candidate for development as a therapeutic for alleviating inflammation.
\end{abstract}

Correspondence to: Professor Sayeon Cho, Laboratory of Molecular and Pharmacological Cell Biology, College of Pharmacy, Chung-Ang University, 84 Heukseok-Ro, Dongjak-Gu, Seoul 06974 , Republic of Korea

E-mail: sycho@cau.ac.kr

Present address: ${ }^{2}$ College of Pharmacy, Chonnam National University, Gwangju 61186, Republic of Korea

*Contributed equally

Key words: Guettarda speciosa Linn., macrophage, lipopolysaccharide, inflammatory mediators, nuclear factor- $\mathrm{kB}$, Syk

\section{Introduction}

Inflammation is a complex immunological response that protects the body from infection by bacteria, viruses and fungi. Inflammatory responses are self-limited through the balance between the inhibition of proinflammatory proteins and the upregulation of anti-inflammatory proteins $(1,2)$. Macrophages are the essential first line of defense against common pathogens. Among the various stimuli of macrophages, lipopolysaccharide (LPS) is an inflammatory stimulator that functions through toll-like receptor 4 (TLR4) on the macrophage membrane surface $(3,4)$. Macrophages activated by LPS produce several proinflammatory mediators, such as nitric oxide (NO), prostaglandin $\mathrm{E}_{2}\left(\mathrm{PGE}_{2}\right)$, interleukin (IL)- 6 and $-1 \beta$, and tumor necrosis factor (TNF)- $\alpha(5,6)$. The enhanced production of inflammatory mediators is necessary for the host defense against external stimuli; however, this is reportedly involved in several inflammatory diseases, including rheumatoid arthritis, systemic lupus erythematosus and inflammatory bowel disease $(7,8)$. Therefore, agents that inhibit excessive production of inflammatory mediators in activated macrophages may be candidates for the treatment of inflammatory diseases.

Guettarda speciosa Linn. (G. speciosa), a member of the Rubiaceae family, is widely distributed from East Africa to South Asia. In East Africa, the stem of G. speciosa is used in a preparation for the treatment of maternal postpartum infection (9). In Tahiti, G. speciosa has been used in anti-diarrheic, febrifugal and anti-cholinergic treatments (10). In addition, the decoction of $G$. speciosa leaves has been used to treat cough, cold, sore throats, fever, dysentery and headache (10). The anti-inflammatory effects of the extracts from $G$. speciosa on intestinal mucosa inflammation in mice have been previously demonstrated $(10,11)$. Several studies have reported a positive correlation between the effects of the therapeutic use of drugs isolated from plants and of the traditional use of the plant extracts from which they are derived $(12,13)$. The phytochemical composition of G. speciosa comprises phenolic components, including squalene, cladosporide $\mathrm{A}$, nonacosane and campesterol, and steroidal components, such as stigmasterol (14). Certain major active components, including squalene, campesterol and stigmasterol, have demonstrated 
anti-inflammatory, anti-cancer, anti-bacterial and anti-oxidant effects (15-17).

Although G. speciosa has been used as a folk medicine and several of its pharmacological activities have been evaluated, the anti-inflammatory mechanisms of this plant have remained to be determined. In the present study, the anti-inflammatory effects of the methanol extract of G. speciosa (MGS) in LPS-activated RAW 264.7 macrophages and the underlying mechanisms of action were investigated.

\section{Materials and methods}

MGS preparation. A methanol extract of $G$. speciosa (Rubiaceae) (voucher no: PBID 11237) collected from Indonesia was purchased from the International Biological Material Research Center (Korea Research Institute of Bioscience and Biotechnology, Daejeon, Korea) (18). The extract was dissolved in dimethyl sulfoxide (DMSO; Sigma-Aldrich; Merck KGaA, Darmstadt, Germany) and added to the culture media to achieve the final concentration as described in each assay. In all experiments, the DMSO concentrations did not exceed $0.1 \%$.

Cell culture and reagents. RAW 264.7 macrophages were purchased from the American Type Culture Collection (Manassas, VA, USA). RAW 264.7 cells were cultured in Dulbecco's modified Eagle's medium (DMEM; Invitrogen; Thermo Fisher Scientific, Inc., Waltham, MA, USA) supplemented with $10 \%$ fetal bovine serum (FBS, Invitrogen; Thermo Fisher Scientific, Inc.), and $1 \%$ penicillin/streptomycin (Thermo Fisher Scientific, Inc.) at $37^{\circ} \mathrm{C}$ in a humidified atmosphere containing $5 \% \mathrm{CO}_{2}$. Mouse polyclonal anti-p38 (cat no. sc-7972), mouse monoclonal anti-c-Jun N-terminal kinase (JNK; cat no. sc-7345), rabbit polyclonal anti-inhibitor

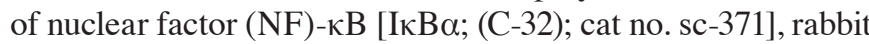
polyclonal phospho (p)-anti-IкB $\alpha$ (Ser32/36; cat no. sc-101713), mouse polyclonal anti-spleen tyrosine kinase (Syk; cat no. sc-1240), mouse monoclonal anti-c-proto-oncogene tyrosine-protein kinase $\mathrm{Src}$ (c-Src cat no. sc-19), rabbit polyclonal anti-p-c-Src (Tyr424; cat no. sc-81521), rabbit polyclonal anti-Akt1/2/3 (cat no. sc-8312), rabbit polyclonal anti-p-Akt1/2/3 (Ser473; cat no. sc-7985), goat polyclonal anti-cyclooxygenase 2 (COX-2; cat no. sc-1745), rabbit polyclonal anti-inducible NO synthase (iNOS; cat no. 651) and mouse monoclonal anti- $\alpha$-tubulin (cat no. sc-5286) antibodies were purchased from Santa Cruz Biotechnology, Inc. (Dallas, TX, USA). Rabbit polyclonal transforming growth factor- $\beta$-activated kinase 1 (TAK1; cat no. 4505), rabbit monoclonal anti-p-TAK1 (Thr184/187; cat no. 4508), rabbit polyclonal anti-mitogen-activated protein kinase (MAPK) kinase 4 (MKK4; cat no. 9152), rabbit polyclonal anti-MKK7 (cat no. 9264), rabbit polyclonal anti-p-MKK4 (Thr261; cat no. 9151), rabbit polyclonal anti-p-MKK7 (Ser217/Thr275; cat no. 4171), rabbit polyclonal anti-p-p38 (Thr180/Tyr182; cat no. 9211), rabbit polyclonal anti-extracellular signal-regulated kinase (ERK; cat no. 9102), rabbit monoclonal anti-p-ERK (Thr202/Tyr204; cat no. 9106), rabbit polyclonal anti-p-JNK (Thr183/Tyr185; cat no. 9252) and rabbit polyclonal anti-p-Syk (Tyr525/526; cat no. 2711) antibodies were purchased from Cell Signaling Technology (Danvers, MA,
USA). All primary antibodies were diluted at 1:1,000 in 5\% non-fat dried milk. Polyclonal anti-rabbit IgG-HRP (1:5,000; cat no. LF-SA8002) and polyclonal anti-mouse IgG Fc-HRP (1:5,000; cat no. LF-SA8001) were from AbFrontier (Young In Frontier Co., Ltd., Seoul, Korea). Ready-SET-Go! ELISA kits were used for the detection of IL-6 (cat no. 88-7084) and TNF- $\alpha$ (cat no. 88-7324) were from eBioscience (Thermo Fisher Scientific, Inc).

Cell viability assay. RAW 264.7 macrophages were seeded in 96-well plates $\left(4.5 \times 10^{4}\right.$ cells/well) and treated with various concentrations of MGS $(50,100,200,300$ and $600 \mu \mathrm{g} / \mathrm{ml})$ and LPS $(1 \mu \mathrm{g} / \mathrm{ml})$ at $37^{\circ} \mathrm{C}$ for $24 \mathrm{~h}$. The cytotoxic effects were evaluated using the EZ-Cytox cell viability assay kit (cat no. EZ-3000, Daeil Lab, Seoul, Korea). EZ-Cytox solution, which contained a water-soluble tetrazolium salt, was added to the cell culture (1/10 culture medium), followed by incubation for $1 \mathrm{~h}$ at $37^{\circ} \mathrm{C}$. The relative absorbance were measured at $450 \mathrm{~nm}$ (absorbance for viable cells) and $650 \mathrm{~nm}$ (reference absorbance) with a Synergy H1 microplate reader (BioTek Instruments, Inc., Winooski, VT, USA).

Nitrite assay. RAW 264.7 macrophages were seeded in 96-well plates at a density of $4.5 \times 10^{4}$ cells/well and incubated at $37^{\circ} \mathrm{C}$ overnight. Subsequently, the cells were incubated with various concentrations of MGS $(50,100,200$ and $300 \mu \mathrm{g} / \mathrm{ml})$ and LPS $(1 \mu \mathrm{g} / \mathrm{ml})$ at $37^{\circ} \mathrm{C}$ for $24 \mathrm{~h}$. Culture media $(100 \mu \mathrm{l})$ was transferred to a new 96 well-plate and $100 \mu \mathrm{l}$ Griess reagent [1\% sulfanilamide, $2.5 \%$ phosphoric acid $\left(\mathrm{H}_{3} \mathrm{PO}_{4}\right)$, and $0.1 \%$ $\mathrm{N}$-(1-naphthyl) ethylenediamine in distilled water] was added. Sodium nitrite was used to generate a standard curve. After incubation at $37^{\circ} \mathrm{C}$ for $10 \mathrm{~min}$, the absorbance was measured at $540 \mathrm{~nm}$ using a Synergy $\mathrm{H} 1$ microplate reader.

ELISA. RAW 264.7 macrophages were treated with various concentrations of MGS (50, 100, 200 and $300 \mu \mathrm{g} / \mathrm{ml})$ and LPS $(1 \mu \mathrm{g} / \mathrm{ml})$ at $37^{\circ} \mathrm{C}$ for $24 \mathrm{~h}$. After stimulation, the concentrations of TNF- $\alpha$ and IL- 6 in the supernatants were measured using a sandwich ELISA with monoclonal antibodies specific for each mediator in accordance with the manufacturer's protocol. Prior to the application of samples, the plate was pre-coated with the capture antibody in the supplied buffer. Following incubation overnight at $4^{\circ} \mathrm{C}$, the plate was washed with $1 \mathrm{X}$ PBS with $0.05 \%$ Tween 20 (PBST) and blocked with $1 \mathrm{X}$ assay diluents at room temperature for $1 \mathrm{~h}$. The solutions were added to each well and incubated for $2 \mathrm{~h}$ at room temperature. The plate was washed with $1 \mathrm{X}$ PBST and treated with a biotinylated detection antibody solution at room temperature for $1 \mathrm{~h}$, which was followed by treatment with a horseradish peroxidase-streptavidin solution at room temperature for $30 \mathrm{~min}$. After one further wash, 3,3',5,5'-tetramethylbenzidine was added and the plate was incubated at room temperature for $10 \mathrm{~min}$ in the dark. Subsequently, $1 \mathrm{~N} \mathrm{H}_{3} \mathrm{PO}_{4}$ was added and the absorbance of individual wells was measured at $450 \mathrm{~nm}$ using a Synergy $\mathrm{H} 1$ microplate reader.

Reverse transcription-quantitative polymerase chain reaction ( $R T-q P C R)$ analysis. RAW 264.7 macrophages were treated with various concentrations of $\operatorname{MGS}(50,100$, 200 and $300 \mu \mathrm{g} / \mathrm{ml})$ and LPS $(1 \mu \mathrm{g} / \mathrm{ml})$ at $37^{\circ} \mathrm{C}$ for $3 \mathrm{~h}$. Total 
RNA was isolated from the cells using Accuzol total RNA extraction solution (Bioneer Corp., Daejeon, Korea) and was reverse transcribed into complementary DNA (cDNA) by using a TOPscript cDNA synthesis kit in accordance with the manufacturer's protocol (Enzynomics, Daejeon, Korea). The amplification of cDNA was performed using a RT-qPCR premix and iTaq Universal SYBR Green Master Mix (Bio-Rad Laboratories, Inc., Hercules, CA, USA) in accordance with the manufacturer's protocol. The PCR was run for 40 cycles of denaturation at $95^{\circ} \mathrm{C}(10 \mathrm{sec})$ and annealing/extension at $55^{\circ} \mathrm{C}$ (30 sec) in a CFX Connect real-time thermal cycler (Bio-Rad Laboratories, Inc.). The gene expressions were quantified using the $2^{-\triangle \Delta C q}$ method, and normalized to the reference genes $\beta$-actin and GAPDH (19). PCR primer sequences used in this study were as follows: Mouse iNOS (forward, 5'-TGGCCA CCAAGCTGAACT-3' and reverse, 5'-TCATGATAACGT TTCTGGCTCTT-3'); COX-2 (forward, 5'-GATGCTCTTCCG AGCTGTG-3' and reverse, 5'-GGATTGGAACAGCAAGGA TTT-3'), TNF- $\alpha$ (forward, 5-CTGTAGCCCACGTCGTAG C-3' and reverse, 5'-TTGAGATCCATGCCGTTG-3'), IL-6 (forward, 5'-TCTAATTCATATCTTCAACCAAGAGG-3' and reverse, 5'-TGGTCCTTAGCCACTCCTTC-3'), IL-1 $\beta$ (forward, 5'-TTGACGGACCCCAAAAGAT-3' and reverse, 5'-GATGTGCTGCTGCGAGATT-3'), $\beta$-actin (forward, 5'-CGTCATACTCCTGCTTGCTG-3' and reverse, 5'-CCA GATCATTGCTCCTCCTGA-3') and GAPDH (forward, 5'-GCTCTCTGCTCCTCCTGTTC-3' and reverse, 5'-ACG ACCAAATCCGTTGACTC-3').

Western blot analysis. RAW 264.7 macrophages were pre-treated with MGS (50, 100, 200 and $300 \mu \mathrm{g} / \mathrm{ml})$ for $2 \mathrm{~h}$ at $37^{\circ} \mathrm{C}$ and stimulated with LPS $(1 \mu \mathrm{g} / \mathrm{ml})$ at $37^{\circ} \mathrm{C}$ for the indicated times to specifically detect the target proteins: $3 \mathrm{~min}$ (for $\mathrm{I} \kappa \mathrm{B} \alpha, \mathrm{Src}$, Syk and Akt), $15 \mathrm{~min}$ (for MAPKs) or $24 \mathrm{~h}$ (for iNOS and COX-2). Cells were washed twice with cold PBS (pH 7.4) and lysed in lysis buffer [150 mM NaCl, $20 \mathrm{mM}$ Tris- $\mathrm{HCl}$ (pH 8.0), 0.5\% IGEPAL ${ }^{\circledR}$ CA-630, 0.5\% Triton X-100, 1 mM EDTA, $1 \%$ glycerol, $2 \mathrm{mM}$ phenylmethylsulfonyl fluoride, $10 \mathrm{mM} \mathrm{NaF}$ and $\left.1 \mathrm{mM} \mathrm{Na}_{3} \mathrm{VO}_{4}\right]$. The lysates were centrifuged at $15,814 \mathrm{x}$ g for $30 \mathrm{~min}$ at $4^{\circ} \mathrm{C}$ and the supernatants were transferred to a new tube. The protein concentration was determined by a Bradford protein assay (Bio-Rad Laboratories, Inc.) in accordance with the manufacturer's protocol and immunoblotting was performed as described previously (20). In brief, equal amounts (30 $\mu \mathrm{g}$ per lane) of protein were mixed with $5 \mathrm{X}$ SDS sample buffer $[12 \mathrm{mM}$ Tris- $\mathrm{HCl}$ (pH 6.8), 0.4\% SDS, $5 \%$ glycerol, $1 \% \beta$-mercaptoethanol and $0.02 \%$ bromophenol blue] and boiled at $100^{\circ} \mathrm{C}$ for $5 \mathrm{~min}$. Samples were separated by $10 \%$ SDS-PAGE and transferred onto nitrocellulose membranes. Membranes were blocked with 5\% nonfat-dried skimmed milk for $1 \mathrm{~h}$ at room temperature in $1 \mathrm{X}$ Tris-buffered saline with $0.05 \%$ Tween 20 (TBST) and each membrane was incubated at $4^{\circ} \mathrm{C}$ overnight with a specific primary antibody. Membranes were washed four times with TBST and then incubated with the appropriate secondary antibody for $2 \mathrm{~h}$ at room temperature. Protein bands were visualized by application of enhanced chemiluminescence immunoblotting detection reagent (Pierce; Thermo Fisher Scientific, Inc.) and protein levels were quantified using LabWorks software version 4.6 (UVP Inc., Upland, CA, USA).
Statistical analysis. Values are expressed as the mean \pm standard error of the mean. The differences between experimental conditions were assessed using one-way analysis of variance and Dunnett's multiple-comparisons test, which were computed using Prism 3.0 software (GraphPad Software, Inc., La Jolla, CA,USA). $\mathrm{P}<0.05$ was considered to indicate a statistically significant difference. Data from nine replicates, comprised of three independent experiments with three replicates, were analyzed for each test condition.

\section{Results}

Effect of MGS on viability of LPS-treated or-untreated RAW 264.7 cells. G. speciosa has been used in folk medicine from East Africa to Asia for the treatment of inflammatory diseases. Recently, several components of G. speciosa extract have been reported to exhibit anti-inflammatory, anti-oxidative, anti-microbial or anti-epileptic effects $(10,16)$. However, to the best of our knowledge, systemic studies to investigate the anti-inflammatory effects and precise mechanisms of $G$. speciosa have not been performed. Therefore, the anti-inflammatory effects of MGS in macrophages were explored by performing cell viability assays to determine the non-cytotoxic concentrations of MGS in RAW 264.7 macrophages. As illustrated in Fig. 1A, MGS exerted only a minor or no effect on cell viability compared with the untreated control group at concentrations of $\leq 300 \mu \mathrm{g} / \mathrm{ml}$ in the absence or presence of LPS. However, cytotoxicity was observed at MGS concentrations of $>300 \mu \mathrm{g} / \mathrm{ml}$. Therefore, MGS concentrations of up to $300 \mu \mathrm{g} / \mathrm{ml}$ were used in the subsequent experiments.

Inhibitory effects of MGS on LPS-induced NO production. Under physiological conditions, NO has several functions, including the elimination of bacteria, control of blood pressure and mediation of neurotransmission (21). However, when an inflammatory reaction occurs, NO levels are increased by inducing iNOS in cells and the generated NO exerts dual roles in immunity and inflammatory responses (22). The anti-inflammatory effects of MGS with regard to its capability to suppress NO production in LPS-treated RAW 264.7 cells was evaluated. The production of NO decreased by MGS in LPS-stimulated RAW 264.7 cells in a dose-dependent manner (Fig. 1B). As iNOS is a key enzyme in the production of NO in LPS-stimulated macrophages, RT-qPCR and immunoblotting were employed to assess whether MGS regulated the expression of iNOS at the mRNA and protein levels, respectively. As demonstrated in Fig. 1C, the mRNA expression levels of iNOS were decreased by MGS. In addition, MGS reduced the LPS-induced increases of iNOS protein expression (Fig. 1D). These results demonstrated that MGS reduced NO production in LPS-activated macrophages through the inhibition of iNOS expression.

Effect of MGS on LPS-induced production of proinflammatory mediators. The biosynthesis of $\mathrm{PGE}_{2}$, which is tightly regulated by COX-2, is significantly increased in inflamed tissue, contributing to the development of the cardinal signs of acute inflammation (23). Therefore, the inhibitory effects of MGS on the mRNA and protein expression of COX-2 were investigated. 

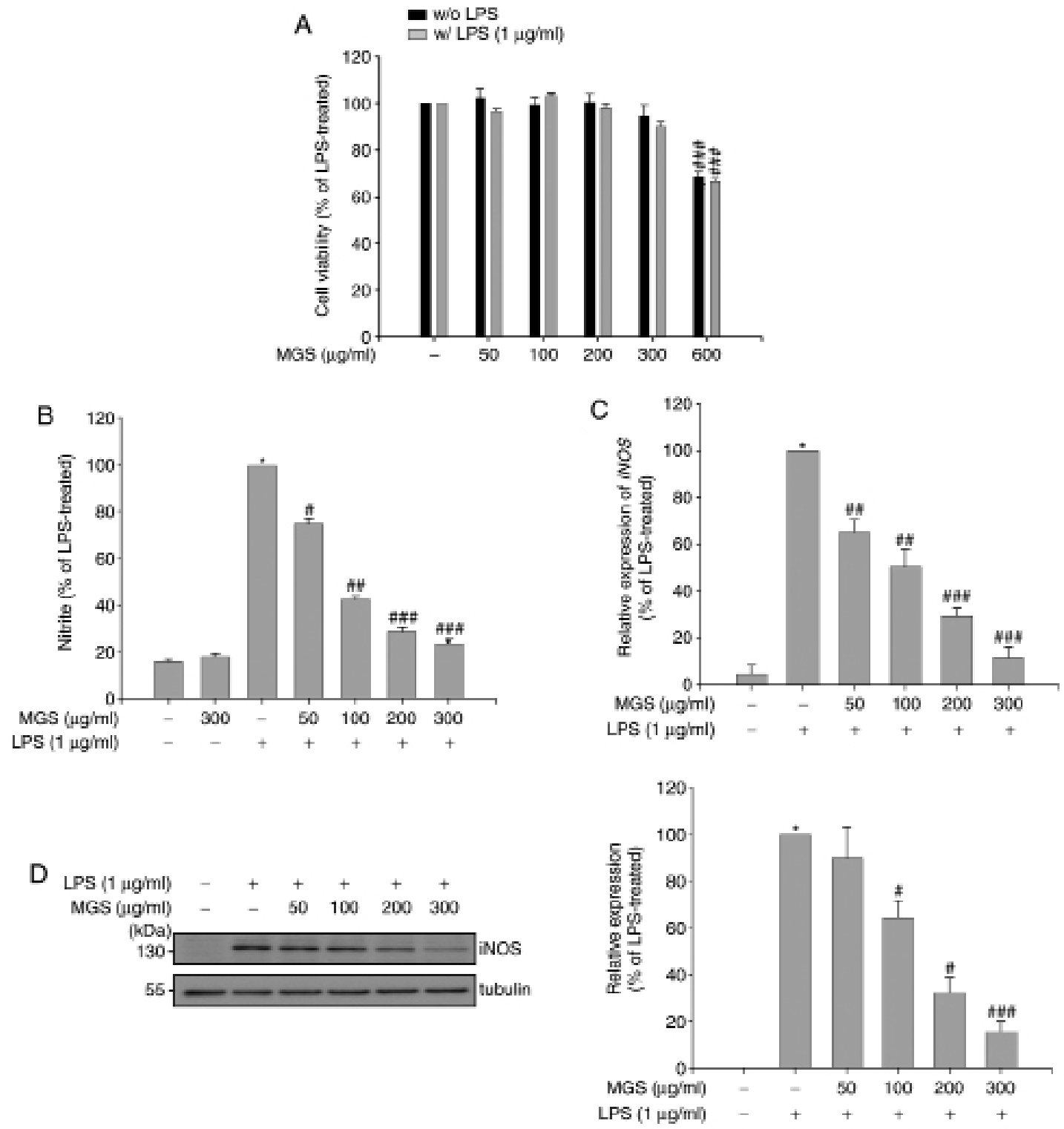

Figure 1. Effects of MGS on cell viability and LPS-mediated NO production. (A) RAW 264.7 macrophages were pretreated with MGS (50, 100, 200, 300, and $600 \mu \mathrm{g} / \mathrm{ml}$ ) for $2 \mathrm{~h}$, followed by incubation with or without LPS and the cell viability was measured using the EZ-Cytox assay kit. (B) After stimulation for $24 \mathrm{~h}$, NO levels in the supernatants were measured using the Griess reagent. After stimulation for $3 \mathrm{~h}$, the iNOS (C) mRNA and (D) protein expression levels in each group were compared to those in the LPS-treated group using reverse-transcription quantitative polymerase chain reaction analysis or western blot analysis with normalization to $\alpha$-tubulin, respectively. Values are expressed as the mean \pm standard error of the mean. "P<0.01 vs. LPS-untreated or LPS-treated control group; ${ }^{\#} \mathrm{P}<0.05,{ }^{\# \#} \mathrm{P}<0.01$ and ${ }^{\# \# \#} \mathrm{P}<0.001$ relative to the LPS-treated group. NO, nitric oxide; iNOS, inducible NO synthase; LPS, lipopolysaccharide; MGS, methanol extract of $G$. speciosa.

In contrast to the reduction of iNOS mRNA expression, MGS did not affect the mRNA expression of COX-2 (Fig. 2A). Similarly, no significant inhibition of LPS-induced COX-2 protein expression by MGS was observed (Fig. 2B). These results suggested that MGS suppressed the expression of iNOS, but not COX-2, in macrophages.

Proinflammatory cytokines are predominantly produced by activated macrophages and are involved in the upregulation of inflammatory reactions (24). There is evidence that certain proinflammatory cytokines, including IL-1 $\beta$, IL- 6 and TNF- $\alpha$, are involved in the mechanisms of pathological pain (25). Therefore, the inhibitory effect of MGS on cytokine production in LPS-stimulated macrophages was tested to further investigate the anti-inflammatory actions of MGS. As presented in Fig. 2C and D, LPS-stimulated RAW 264.7 macrophages produced large amounts of the cytokines IL- 6 and TNF- $\alpha$. MGS treatment reduced the LPS-stimulated production of IL-6, but did not change TNF- $\alpha$ production. To determine whether the effect of MGS on the production of proinflammatory cytokines was regulated at the transcriptional level, the mRNA expression of proinflammatory cytokines was assessed in LPS-stimulated RAW 264.7 cells after treatment with MGS. RT-qPCR analysis revealed that MGS inhibited the expression of IL-6 mRNA in LPS-stimulated RAW 264.7 cells, but did not inhibit TNF- $\alpha$ and IL-1 $\beta$ mRNA levels (Fig. 2E). These results indicated that MGS selectively regulated the production of the proinflammatory cytokine IL-6 at the mRNA and protein level. and NF- $\kappa B$ activation in RAW 264.7 macrophages. Owing to 

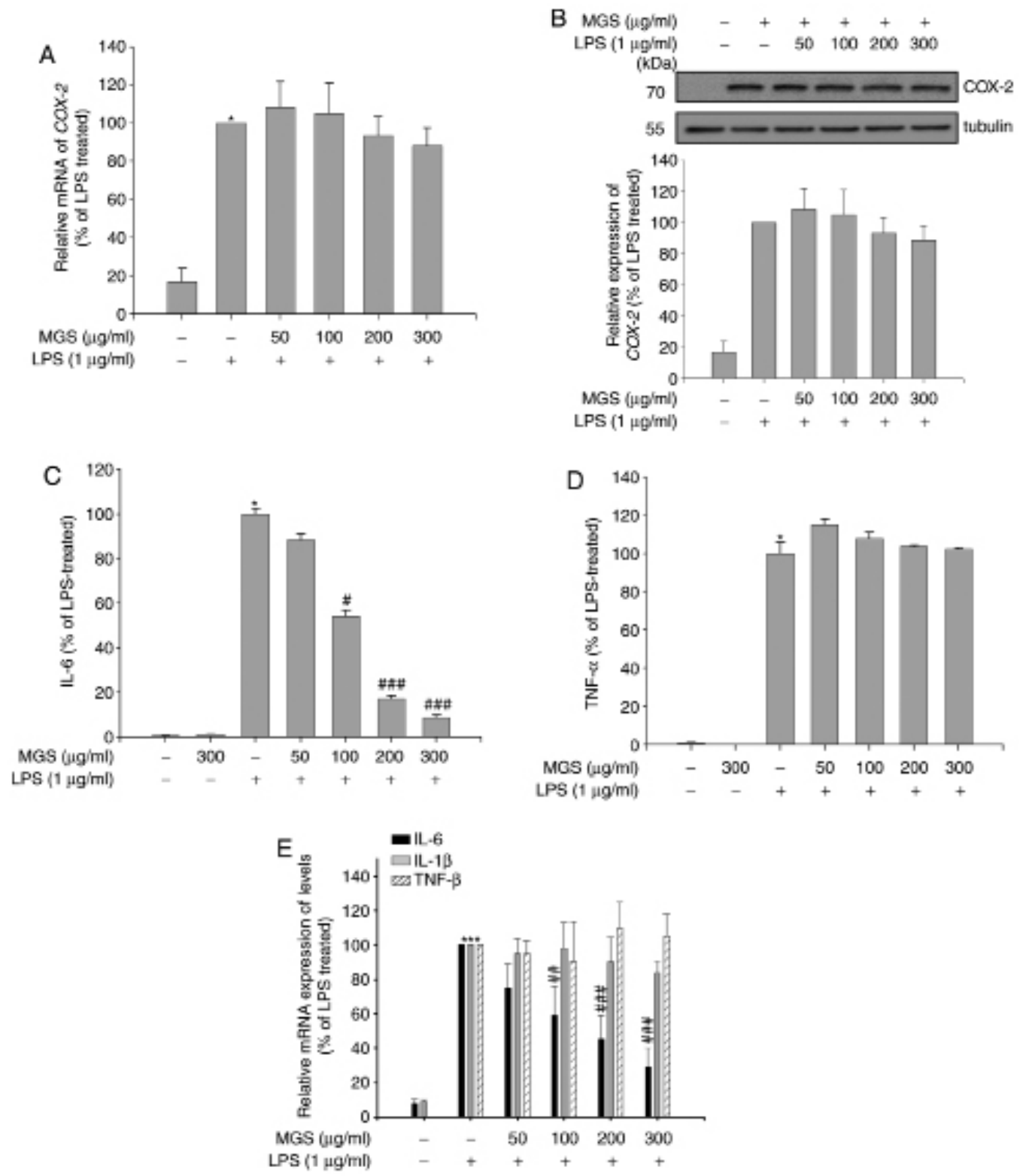

Figure 2. Effect of MGS on the LPS-induced production of proinflammatory mediators. RAW 264.7 macrophages were pretreated with MGS (50, 100, 200, and $300 \mu \mathrm{g} / \mathrm{ml}$ ) for $2 \mathrm{~h}$ and stimulated with LPS for the indicated times. After stimulation for $3 \mathrm{~h}$, COX2 (A) mRNA and (B) protein expression levels were measured in each group using RT-qPCR analysis or western blot analysis with normalization to $\alpha$-tubulin, respectively. After stimulation for $24 \mathrm{~h}$, protein expression levels of (C) IL-6 and (D) TNF- $\alpha$ were measured by ELISA and (E) relative mRNA expression levels of IL-1 $\beta$, IL- 6 and TNF- $\alpha$ were measured by RT-qPCR. Values are expressed as the mean \pm standard error or the mean. ${ }^{*} \mathrm{P}<0.01$ vs. LPS-untreated group; ${ }^{*} \mathrm{P}<0.05,{ }^{\# \#} \mathrm{P}<0.01$ and ${ }^{\# \# "} \mathrm{P}<0.001$ relative to the LPS-treated group. COX, cyclooxygenase; IL, interleukin; LPS, lipopolysaccharide; MGS, methanol extract of G. speciosa; TNF, tumor necrosis factor; RT-qPCR, reverse transcription-quantitative polymerase chain reaction.

the relevance of the MAPK and NF- $\mathrm{kB}$ signaling pathways in LPS-induced cytokine gene expression in inflammation (26,27), the modulatory effect of MGS on these pathways in macrophages was investigated. The transcription factor $\mathrm{NF}-\kappa \mathrm{B}$ performs critical roles in inflammation, immunity, cell proliferation, differentiation and survival (28). NF- $\mathrm{KB}$ activation depends on phosphorylation of IкB $\alpha$ at Ser32/36; in unstimulated cells, the proteasomal degradation of phosphory-

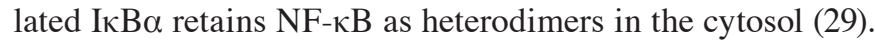
Therefore, the levels of total and $\mathrm{p}-\mathrm{I} \kappa \mathrm{B} \alpha$ were measured to assess the involvement of the NF- $\mathrm{BB}$ pathway in the anti-inflammatory mechanism of MGS in LPS-induced macrophages. As demonstrated in Fig. 3A, LPS treatment increased the phosphorylation of IкB $\alpha$ and reduced the levels of total, unphosphorylated I $\mathrm{I} \mathrm{B} \alpha$; however, in the MGS-treated groups, the LPS-induced phosphorylation of I $\mathrm{B} \alpha$ and reduction of its expression were decreased.

Studies have demonstrated that MAPKs respond to extraand intracellular stimuli and regulate immune responses, including proinflammatory cytokine production, as well as cell proliferation, differentiation and survival $(30,31)$. In the present study, the suppression of the phosphorylation status in the activation loops of MAPKs was examined by immunoblotting analysis to identify the role of MGS in MAPK signaling pathways. As illustrated in Fig. 3B, the phosphorylation of all MAPKs was induced in LPS-stimulated RAW 264.7 macrophages. However, only the LPS-induced phosphorylation of JNK was moderately inhibited after MGS treatment; that of 

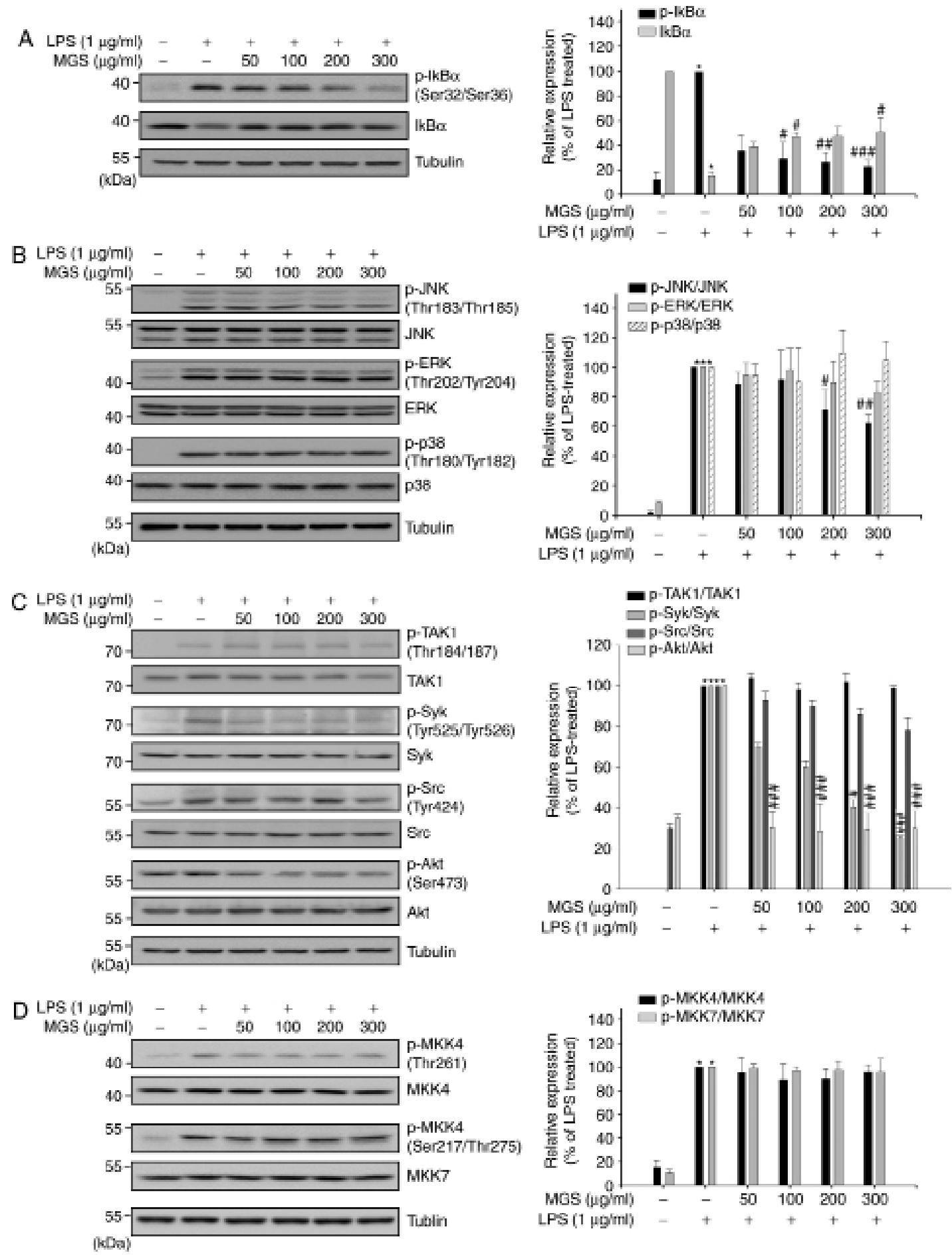

Figure 3. Selective inhibition of MGS on MAPK phosphorylation and NF-kB activation in RAW 264.7 macrophages. RAW 264.7 macrophages were pretreated with MGS $(50,100,200$ and $300 \mu \mathrm{g} / \mathrm{ml})$ for $2 \mathrm{~h}$ and stimulated with LPS for indicated times. (A) After stimulation for 3 min, protein expression levels of $\mathrm{p}-\mathrm{I} \kappa \mathrm{B} \alpha$ and Iк $\mathrm{B} \alpha$ were measured by western blot analysis. (B) After stimulation for 15 min, protein expression levels of JNK, p-JNK, ERK, p-ERK, p38 and p-p38 were measured by western blot analysis. (C) After stimulation for 3 min, protein expression levels of TAK1, p-TAK1, Syk, p-Syk, Src, p-Src, Akt and p-Akt and (D) after stimulation for 15 min, protein expression levels of MKK4, p-MKK4 and-7 were measured by western blot analysis. Values are expressed as the mean \pm standard error of the mean. ${ }^{*} \mathrm{P}<0.01$ vs. LPS-untreated group; ${ }^{\# \mathrm{P}}<0.05,{ }^{\# \#} \mathrm{P}<0.01$ and ${ }^{\# \# \#} \mathrm{P}<0.001$ relative to the LPS-treated group. ERK, extracellular signal-regulated kinase; JNK, c-Jun N-terminal kinase; LPS, lipopolysaccharide; MGS, methanol extract of G. speciosa; MKK, mitogen-activated protein kinase kinase; p, phosphorylated; Src, sarcoma; Syk, spleen tyrosine kinase; IкB, inhibitor of nuclear factor $\kappa$ B.

ERK1/2 and p38 was not changed. As the phosphorylation status of MAPKs is directly associated with their kinase activity (32), the results suggested that MGS reduced the production of proinflammatory mediators through the inhibition of NF-kB and JNK signaling.
In addition, Syk/Src/Akt signaling pathways have a key role in NF- $\mathrm{KB}$ activation, whereas JNK activation is induced by MKK4/7 $(33,34)$. To clarify the target of MGS in the regulation of NF- $\mathrm{KB}$ and JNK signaling pathways, the effects of MGS on the phosphorylation of Syk/Src/Akt and MKK4/7 
were determined. The LPS-increased phosphorylation of Syk and Akt was decreased by MGS treatment (Fig. 3C). However, the LPS-induced phosphorylation of MKK4/7 did not appear to be affected by MGS treatment (Fig. 3D). In conclusion, these results suggested that, in RAW 264.7 macrophages, MGS regulated $\mathrm{NF}-\kappa \mathrm{B}$ activity through the inhibition of the Syk/Akt axis and that its inhibitory effect on JNK activation did not proceed via the regulation of MKK4/7.

\section{Discussion}

The production of NO by iNOS after certain stimuli is one of the most important steps in the inflammatory process (35). Numerous studies have attempted to identify novel anti-inflammatory agents that inhibit iNOS expression and elicit their mechanisms of action in inflammation (36,37). For instance, COX-2, which ultimately induces inflammation and fever, catalyzes the production of $\mathrm{PGE}_{2}$ from the lipid arachidonic acid (38). The results of the present study demonstrated that MGS inhibited the expression of iNOS, but did not suppress COX-2 expression. Previous studies have reported that the selective regulation of proinflammatory signaling pathways by anti-inflammatory extracts led to the differential inhibition of proinflammatory mediators $(39,40)$. Based on the results of the present study and those of previous reports, the selective inhibitory effects of MGS on the production of proinflammatory mediators are likely to be a result of MGS-mediated selective inhibition of certain associated upstream signaling pathways.

MGS reduced the production of IL- 6 , but not IL-1 $\beta$ and TNF- $\alpha$, in LPS-induced RAW 264.7 cells. Although the promoter region of each proinflammatory cytokine is known to contain binding sites for $\mathrm{NF}-\kappa \mathrm{B}$ and downstream transcription factors of MAPKs, previous studies by our group demonstrated that extracts of natural plants exert different inhibitory effects on the production of proinflammatory cytokines as a result of differential regulation of NF- $\kappa B$ and MAPKs (41). Of note, the inhibition of TNF- $\alpha$ production was only detected when the extract inhibited NF- $\kappa \mathrm{B}$ and all MAPKs, including JNK, ERK and p38 (42). In one instance, TNF- $\alpha$ production was not attenuated when the extract only inhibited one MAPK in addition to NF- $\mathrm{B}$ (43). Therefore, it may be speculated that the MGS-mediated selective inhibition of cytokine production is a consequence of the selective regulation of inflammatory signal transduction, but further studies are needed to confirm the above.

In the present study, MGS suppressed the LPS-induced phosphorylation of I $\mathrm{B} \alpha$ and JNK. The MGS-mediated dephosphorylation of TAK1 was measured, as TAK1 is a well-known upstream kinase in the activation of $N F-\kappa B$ and MAPKs, including JNK and p38 (44). However, no significant inhibition of TAK1 phosphorylation was detected by MGS treatment (Fig. 3C). Previous studies to determine the regulatory effects of MGS on the upstream signaling molecules of I $\mathrm{B} \alpha$ and JNK revealed that MGS regulated the phosphorylation of Syk/Akt, upstream signaling proteins of $\mathrm{NF}-\kappa \mathrm{B}$ activation, whereas MKK4 and MMK7, the specific upstream signaling proteins of JNK, were not affected by MGS treatment. Further studies, including those on the measurement of

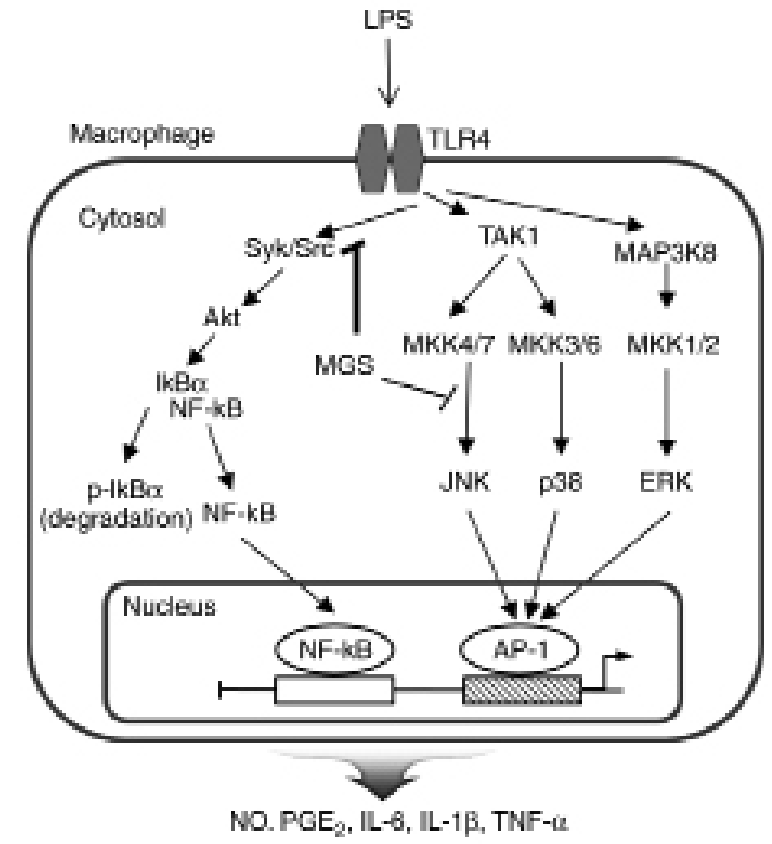

Figure 4. Schematic representation of the proposed anti-inflammatory mechanism of MGS in LPS-activated RAW 264.7 macrophages. MGS may inhibit the inflammatory mediators via regulation of the NF- $\mathrm{KB}$ and JNK signaling pathways. The molecules and inflammatory mediators regulated by MGS are stated in bold letters. JNK, c-Jun N-terminal kinase; LPS,

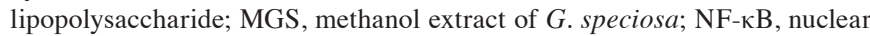
factor $\kappa \mathrm{B}$; MAPK, mitogen-activated protein kinase; MKK, MAPK kinase; I $\kappa$ B, inhibitor of NF- $\kappa \mathrm{B}$; TLR, Toll-like receptor; AP-1, activator protein 1; ERK, extracellular signal-regulated kinase; p, phosphorylated; Src, sarcoma; Syk, spleen tyrosine kinase; TAK1, transforming growth factor- $\beta$-activated kinase 1; NO, nitric oxide; LPS, lipopolysaccharide; TNF, tumor necrosis factor; PGE2, prostaglandin $\mathrm{E}_{2}$.

MKK4/7 kinase activity after MGS treatment, are needed to clarify the underlying mechanism(s) of the regulatory effect of MGS on JNK activation.

Several studies have reported that the anti-inflammatory properties of the individual active components define the anti-inflammatory functions of an extract and its signal regulatory ability $(36,45)$. Phytochemical analysis of MGS performed by gas chromatography tandem mass spectrometry demonstrated that MGS contained several anti-inflammatory components, including squalene, campesterol and stigmasterol, that are known to selectively regulate inflammatory responses in different ways (14). Squalene was reported to inhibit inflammatory responses in macrophages via the inhibition of JNK and NF- $\kappa \mathrm{B}$ pathways (15). Campesterol and stigmasterol inhibit the production of inflammatory mediators via the suppression of the $N F-\kappa B$ signaling pathway in chondrocytes $(16,46,47)$. It may be proposed that the selective inhibition of $\mathrm{NF}-\kappa \mathrm{B}$ and JNK pathways by MGS is a result of the regulatory effects of its anti-inflammatory components on these signaling pathways, but further studies are needed to identify specific roles of the components (Fig. 4).

In conclusion, MGS inhibited NO and IL-6 production through the suppression of certain molecular signaling molecules associated with the inflammatory response, including $\mathrm{NF}-\kappa \mathrm{B}$ and $\mathrm{JNK}$ activation. Therefore, owing to the observed anti-inflammatory effect of MGS, this 
extract may be considered for the development of an effective anti-inflammatory agent for the treatment of severe inflammation.

\section{Acknowledgements}

The present study was supported by the National Research Foundation of Korea (NRF) with funding from the Ministry of Science, ICT \& Future Planning (grant nos. NRF-2015R1A2A2A11001446 and NRF-2015R1A5A1008958).

\section{References}

1. Abdalla SI, Sanderson IR and Fitzgerald RC: Effect of inflammation on cyclooxygenase (COX)-2 expression in benign and malignant oesophageal cells. Carcinogenesis 26: 1627-1633, 2005 .

2. Franchi J, Marteau C, Crola da Silva C, Mitterrand M, André P and Kieda C: Cell model of inflammation. Biosci Rep 28: 23-32, 2008.

3. Billack B: Macrophage activation: Role of toll-like receptors, nitric oxide, and nuclear factor kappa B. Am J Pharm Educ 70: 102,2006

4. Takeda $\mathrm{K}$ and Akira S: Toll-like receptors in innate immunity. Int Immunol 17: 1-14, 2005.

5. Lee KJ, Kim YK, Krupa M, Nguyen AN, Do BH, Chung B, Vu TT, Kim SC and Choe H: Crotamine stimulates phagocytic activity by inducing nitric oxide and TNF- $\alpha$ via p38 and NF $\kappa-B$ signaling in RAW 264.7 macrophages. BMB Rep 49: 185-190, 2016.

6. Meng F and Lowell CA: Lipopolysaccharide (LPS)-induced macrophage activation and signal transduction in the absence of Src-family kinases Hck, Fgr, and Lyn. J Exp Med 185: 1661-1670, 1997.

7. Evans SS, Repasky EA and Fisher DT: Fever and the thermal regulation of immunity: The immune system feels the heat. Nat Rev Immunol 15: 335-349, 2015.

8. Juhn SK, Jung MK, Hoffman MD, Drew BR, Preciado DA, Sausen NJ, Jung TT, Kim BH, Park SY, Lin J, et al: The role of inflammatory mediators in the pathogenesis of Otitis media and Sequelae. Clin Exp Otorhinolaryngol 1: 117-138, 2008.

9. Cai WH, Matsunami K, Otsuka H, Shinzato T and Takeda Y: A glycerol $\alpha$-D-glucuronide and a megastigmane glycoside from the leaves of Guettarda speciosa L. J Nat Med 65: 364-369, 2011.

10. Gandhimathi R, Saravana KA, Senthil Kumar KK, Kusuma PK and Uma MJ: Pharmacological studies of anti-diarrhoeal activity of Guettarda speciosa (L.) in experimental animals. J Pharm Sci Res 2: 61-67, 2009.

11. Saravana KA and Gandhimathi R: Effect of Guettarda speciosa extracts on antioxidant enzymes levels in rat brain after induction of seizures by MES and PTZ. J Nat Prod 3: 80-85, 2010.

12. Atanasov AG, Waltenberger B, Pferschy-Wenzig EM, Linder T, Wawrosch C, Uhrin P, Temml V, Wang L, Schwaiger S, Heiss EH, et al: Discovery and resupply of pharmacologically active plant-derived natural products: A review. Biotechnol Adv 33: 1582-1614, 2015.

13. Oliveira AB, Dolabela MF, Braga FC, Jácome RL, Varotti FP and Póvoa MM: Plant-derived antimalarial agents: New leads and efficient phythomedicines. Part I. Alkaloids. An Acad Bras Cienc 81: 715-740, 2009.

14. Revathi D and Rajeswari M: Chemical profiling of Guettarda speciosa Linn. by GC-MS. Int J Adv Res Technol 5, 2015.

15. Cárdeno A, Aparicio-Soto M, Montserrat-de la Paz S, Bermudez B, J.G.Muriana F and Alarcón-de-la-Lastra C: Squalene targets pro- and anti-inflammatory mediators and pathways to modulate over-activation of neutrophils, monocytes and macrophages. J Funct Foods 14: 779-790, 2015.

16. Gabay O, Sanchez C, Salvat C, Chevy F, Breton M, Nourissat G, Wolf C, Jacques C and Berenbaum F: Stigmasterol: A phytosterol with potential anti-osteoarthritic properties. Osteoarthritis Cartilage 18: 106-116, 2010.
17. Sabeva NS, McPhaul CM, Li X, Cory TJ, Feola DJ and Graf GA: Phytosterols differentially influence ABC transporter expression, cholesterol efflux and inflammatory cytokine secretion in macrophage foam cells. J Nutr Biochem 22: 777-783, 2011.

18. Chemat F, Vian MA and Cravotto G: Green extraction of natural products: Concept and principles. Int J Mol Sci 13: 8615-8627, 2012.

19. Livak KJ and Schmittgen TD: Analysis of relative gene expression data using real-time quantitative PCR and the 2(-Delta Delta C(T)) method. Methods 25: 402-408, 2001.

20. Le HTT, Cho YC and Cho S: Inhibition of protein tyrosine phosphatase non-receptor type 2 by PTP inhibitor XIX: Its role as a multiphosphatase inhibitor. BMB Rep 50: 329-334, 2017.

21. Förstermann U and Sessa WC: Nitric oxide synthases: Regulation and function. Eur Heart J 33: 829-837, 2012.

22. Tripathi P, Tripathi P, Kashyap L and Singh V: The role of nitric oxide in inflammatory reactions. FEMS Immunol Med Microbiol 51: 443-452, 2007.

23. Liclican EL, Nguyen V, Sullivan AB and Gronert K: Selective activation of the Prostaglandin E(2) circuit in chronic injury-induced pathologic angiogenesis. Invest Ophthalmol Vis Sci 51: 6311-6320, 2010.

24. Zhang JM and An J: Cytokines, inflammation, and pain. Int Anesthesiol Clin 45: 27-37, 2007

25. Wojdasiewicz P, Poniatowski ŁA and Szukiewicz D: The role of inflammatory and anti-inflammatory cytokines in the pathogenesis of osteoarthritis. Mediators Inflamm 2014: 561459, 2014.

26. Lee JH, Min DS, Lee CW, Song KH, Kim YS and Kim HP: Ginsenosides from Korean Red Ginseng ameliorate lung inflammatory responses: Inhibition of the MAPKs/NF-kB/c-Fos pathways. J Ginseng Res, 2017.

27. Zhang P, Martin M, Michalek SM and Katz J: Role of mitogen-activated protein kinases and NF- $\mathrm{KB}$ in the regulation of proinflammatory and anti-inflammatory cytokines by porphyromonas gingivalis Hemagglutinin B. Infect Immun 73: 3990-3999, 2005.

28. Caamaño $J$ and Hunter $C A$ : $N F-\kappa B$ family of transcription factors: Central regulators of innate and adaptive immune functions. Clin Microbiol Rev 15: 414-429, 2002.

29. Oeckinghaus A and Ghosh S: The NF- $\kappa B$ family of transcription factors and its regulation. Cold Spring Harb Perspect Biol 1: a000034, 2009.

30. Cargnello $M$ and Roux PP: Activation and function of the MAPKs and their substrates, the MAPK-activated protein kinases. Microbiol Mol Biol Rev 75: 50-83, 2011.

31. Muralidharan S and Mandrekar P: Cellular stress response and innate immune signaling: Integrating pathways in host defense and inflammation. J Leukoc Biol 94: 1167-1184, 2013.

32. Whitmarsh AJ: Regulation of gene transcription by mitogen-activated protein kinase signaling pathways. Biochim Biophys Acta 1773: 1285-1298, 2007.

33. Lopez-Bergami P and Ronai Z: Requirements for PKC-augmented JNK activation by MKK4/7. Int J Biochem Cell Biol 40: 1055-1064, 2008.

34. Lowell CA: Src-family and Syk kinases in aActivating and inhibitory pathways in innate immune cells-signaling crosstalk. Cold Spring Harb Perspect Biol 3: a002352, 2011.

35. Miljković D and Spasojević I: Multiple sclerosis: Molecular mechanisms and therapeutic opportunities. Antioxid Redox Signal 19: 2286-2334, 2013.

36. Aggarwal BB, Prasad S, Reuter S, Kannappan R, Yadev VR, Park B, Kim JH, Gupta SC, Phromnoi K, Sundaram C, et al: Identification of novel anti-inflammatory agents from ayurvedic medicine for prevention of chronic diseases: 'Reverse pharmacology' and 'Bedside to bench' approach. Curr Drug Targets 12: 1595-1653, 2011.

37. Gilroy DW: New insights into the anti-inflammatory actions of aspirin-induction of nitric oxide through the generation of epi-lipoxins. Mem Inst Oswaldo Cruz 100 (Suppl 1): S49-S54, 2005.

38. Ricciotti E and FitzGerald GA: Prostaglandins and inflammation. Arterioscler Thromb Vasc Biol 31: 986-1000, 2011.

39. Miyahara K, Murayama H, Wakabayashi H, Kurihara T, Hashimoto K, Satoh K, Motohashi N and Sakagami H: Inhibition of LPS-stimulated NO production in mouse macrophage-like cells by benzocycloheptoxazines. Anticancer Res 28: 2657-2662, 2008 . 
40. Suga A, Narita T, Zhou L, Sakagami H, Satoh K and Wakabayashi H: Inhibition of NO production in LPS-stimulated mouse macrophage-like cells by benzo[b]cyclohept[e][1,4] oxazine and 2-aminotropone derivatives. In Vivo 23: 691-697, 2009.

41. Wullaert A, Bonnet MC and Pasparakis $\mathrm{M}: \mathrm{NF}-\kappa \mathrm{B}$ in the regulation of epithelial homeostasis and inflammation. Cell Res 21: 146-158, 2011.

42. Seong YA, Hwang D and Kim GD: The anti-inflammatory effect of Gnaphalium affine through inhibition of NF- $\kappa B$ and MAPK in lipopolysaccharide-stimulated RAW264.7 cells and analysis of its phytochemical components. Cell Biochem Biophys 74: 407-417, 2016

43. Cho YC and Cho S: c-Jun N-terminal kinase-mediated anti-inflammatory effects of Garcinia subelliptica in macrophages. Mol Med Rep 13: 2293-2300, 2016

44. Sato S, Sanjo H, Takeda K, Ninomiya-Tsuji J, Yamamoto M, Kawai T, Matsumoto K, Takeuchi O and Akira S: Essential function for the kinase TAK1 in innate and adaptive immune responses. Nat Immunol 6: 1087-1095, 2005.
45. Choi RJ, Ngoc TM, Bae K, Cho HJ, Kim DD, Chun J, Khan S and Kim YS: Anti-inflammatory properties of anthraquinones and their relationship with the regulation of P-glycoprotein function and expression. Eur J Pharm Sci 48: 272-281, 2013.

46. Moreno-Anzurez NE, Marquina S, Alvarez L, Zamilpa A, Castillo-España P, Perea-Arango I, Torres PN, Herrera-Ruiz M, Díaz García ER, García JT and Arellano-García J: A cytotoxic and anti-inflammatory campesterol derivative from genetically transformed hairy roots of Lopezia racemosa Cav. (Onagraceae). Molecules 22: E118, 2017.

47. Pandith $\mathrm{H}$, Zhang $\mathrm{X}$, Thong praditchote $\mathrm{S}$, Wongkrajang $\mathrm{Y}$, Gritsanapan W and Baek SJ: Effect of Siam weed extract and its bioactive component scutellarein tetramethyl ether on anti-inflammatory activity through $\mathrm{NF}-\kappa \mathrm{B}$ pathway. J Ethnopharmacol 147: 434-441, 2013. 